\title{
Bipolar disorder associated with interferon-alpha treatment
}

\author{
Iulian Iancu, Anna Sverdlik, Pinhas N Dannon, Elie Lepkifker
}

Interferon-alpha (Hoffman-La Roche Ltd, Basel, Switzerland) is a highly purified, bacterially synthesized agent with antiviral, antiproliferative and immunomodulatory properties. ${ }^{1}$ Interferon-alpha has been approved for the treatment of several types of cancer (eg, leukaemia, lymphoma, renal cell carcinoma and melanoma) and of chronic hepatitis. ${ }^{2,4}$ Usually causing flu-like symptoms, interferonalpha's most serious adverse effects are those related to the central nervous system, including psychiatric side-effects. ${ }^{1,5,6}$ Psychiatric adverse effects include anxiety, irritability, depression, psychosis and delirium ${ }^{1,3,6-9}$ and even suicidality. ${ }^{10}$

We report the precipitation of bipolar disorder after interferon-alpha therapy for chronic myeloid leukaemia in a previously healthy 47 year-old man. We believe that this constitutes the first report of development of bipolar disorder in association with interferon-alpha treatment.

\section{Case report}

A 47-year-old Jewish man of Eastern European descent was referred to our psychiatric emergency room by his haematologist due to insomnia. His parents had divorced when he was 15 years old. He was married and was the father of three children. He had always been introverted with a low self-image. He exhibited learning difficulties and bad social ties. After full-term military service, he worked at different jobs and for the last 10 years he had worked as a container contractor. There was no history of familial psychopathology; he had used flunitrazepam ( $2 \mathrm{mg} /$ day) for sleep induction for the last year. Seven years ago he had developed Graves disease which was treated successfully with mercaptizol. No thyroid dysfunction was noted at follow-up.

One year previously, after a routine blood count revealed leukocytosis, he underwent a bone marrow biopsy and was diagnosed as having chronic myeloid leukaemia (Philadelphia positive). He was asymptomatic and there was no evidence of hepatosplenomegaly or lymph node enlargement. He began treatment with interferon-alpha 9 million units/day, which he had received for seven months until his admission.

While on this treatment, he reported flu-like symptoms and impotence, but continued to function. The haematologist decided to continue the interferon treatment due to partial improvement in the blood cell count. How- ever, in the last three months the patient became gradually euphoric and hyperactive, and also exhibited tension, insomnia and talkativeness. He began to spend more money and bought a great deal of car devices. In the last month his condition worsened and finally he had not slept for a whole week and was very irritable. He felt very self-confident, thought that he was highly sociable, superior, and analytical and that he was going to become rich. $\mathrm{He}$ also showed increased interest in his wife's sexual pleasures.

On admission he was agitated and had a fluent speech, which was difficult to stop. His affect was euphoric and labile. The thought process was circumstantial. He revealed megalomanic thoughts and complimented his doctor on being the greatest psychiatrist in the world! No hallucinations were revealed.

Physical examination was normal (including fundus). Laboratory tests revealed 18000 leucocytes with $80 \%$ polymorphonucleocytes (without signs of chronic myeloid leukaemia); low thyroxine and free thyroxine index and high thyroid-stimulating hormone (another side effect of the interferon-alpha treatment). Brain computed tomography (CT) and electroencephalography (EEG) were normal. A Mini-Mental State Examination revealed normal cognitive functioning (score of 28).

A diagnosis of manic episode with psychotic features was made and the patient was started on perphenazine $32 \mathrm{mg} /$ day and clonazepam $2 \mathrm{mg} /$ day. Gradually, the hyperactivity and insomnia abated. His score on the Brief Psychiatric Rating Scale decreased from an initial score of 50 to 20 after a week $(60 \%$ improvement). His irritability and delusions of superiority gradually abated and after two weeks he stopped the medication and discharged himself against medical advice. Afer a short period of euthymia, he developed a moderate depression which resolved slowly after six months with fluvoxamine $(250 \mathrm{mg}$ / day) and lithium (900 mg/day) therapy.

\section{Discussion}

At admission, the patient had no signs of chronic myeloid leukaemia and his CT and EEG were normal, as was neurological examination. Thus, we believe that the occurence of the manic episode was not related to the chronic myeloid leukaemia. Also, he had not interrupted his benzodiazepine use, ruling out withdrawal as aetiology of the mania. The interruption of interferon-alpha did not lead to 
full recovery, however, so we assume that interferon-alpha precipitated the development of a latent bipolar disorder.

Jannsen et al ${ }^{10}$ reported a short (four week) manic psychosis in a 49-year-old patient treated with interferon-alpha for hepatitis $B$. Van Thiel et al ${ }^{11}$ reported manic exacerbation in two patients with bipolar disorder treated with interferon-alpha for hepatitis $C$. The interruption of interferon-alpha, together with a lithium dose increase, enabled a quick recovery in these patients (Van Thiel, personal communication, 1995).

The exact role of interferon-alpha in the origin of psychiatric side-effects is as yet unknown. Although its passage through the blood-brain barrier is limited due to its being a large hydrophilic protein, interferon-alpha has a direct toxic effect on vasculature and thus might diffuse into the brain. Psychiatric symptoms and EEG abnormalities during therapy have been described in detail. ${ }^{6,10}$ Interferonalpha or one of its metabolites could be directly neurotoxic, but may also induce other substances which interfere with neuronal excitation or neuroendocrine regulation. ${ }^{12,13}$ Patients with brain metastases or other central nervous system abnormalities and those using

1 Jones GJ, Itri LM. Safety and tolerance of recombinant interferon-alpha-2a (Roferon-A) in cancer patients. Cancer 1986; 57: $1709-15$.

2 Taguchi $T$. Clinical studies of recombinant interferon alfa2a (Roferon A) in cancer patients. Cancer 1986; 57: 17058 .

$3 \stackrel{8}{\text { Tsa }}$

Tsavaris N, Mydonakis N, Bacoyiannis C, et al. Treatment of renal cell carcinoma with escalating doses of alphainterferon. Chemotherapy 1993; 39: $361-6$.

4 Rubin RA, Falestiny M, Malet PF. Chronic hepatitis C. Advances in diagnostic testing and therapy. Arch Intern Med 1994; 154: 387-92.

5 Spiegel RJ. The alpha-interferons: clinical overview. Semin Oncol 1987; 14 (suppl 2): $1-12$.

6 Renault PF, Hoofnagle JH, Park Y, et al. Psychiatric complications of long-term interferon alfa therapy. Arch Intern Med 1987; 147: 1577-80.

7 McDonald EM, Mann AH, Thomas HC. Interferons as mediators of psychiatric morbidity. An investigation in a mediators of psychiatric morbidity. An investigation in a
trial of recombinant alpha-interferon in hepatitis B carriers. trial of recombinant alpha 1987 ii: $1175-8$.

\section{Learning points}

- careful monitoring is recommended in patients receiving interferon-alpha with specific attention to mood alterations, psychosis, insomnia and confusion

- high interferon-alpha doses, brain metastases, narcotics use, old age and perhaps psychological lability might render patients susceptible to an increased frequency of psychiatric side-effects

narcotics may experience heightened neurotoxicity. Patients over 40 years old and those receiving doses higher than 6 million units are susceptible to psychiatric side-effects. ${ }^{5}$ These side-effects usually clear within two to three weeks. $^{5}$

As the number of patients treated with interferon-alpha is increasing, its range of psychiatric side-effects should be well recognised. In most cases the management strategy will depend on the extent and severity of the problem, but will usually involve dosage reduction, intermittent breaks in treatment, or psychotropic treatment. ${ }^{1,6-11}$

8 Adams F, Quesada JR, Gutterman JU. Neuropsychiatric manifestations of human leucocyte interferon therapy in matients with cancer. $7 A M A 1984 ; 252$ : $938-41$.

9 patients with cancer. $\mathcal{F} A M A$ 1984; 252: $938-41$. Levenson $\mathrm{JL}$, Fallon H. Fluoxetine treatment of depression
caused by interferon-alfa. Am $\mathcal{f}$ Gastroenterol 1993; 88: $760-1$.

10 Janssen HLA, Brouwer JT, van der Mast RC, Schalm SW. Suicide associated with alfa-interferon therapy for chronic viral hepatitis. $\mathcal{f}$ Hepatol 1994; 21: $241-3$.

11 Van Thiel DH, Friedlander L, Molloy PJ, Fagiuoli S, Kania RJ, Caraceni P. Interferon-alpha can be used successfully in patients with hepatitis $C$ virus-positive chronic hepatitis who have a psychiatric illness. Eur $\mathcal{f}$ Gastroenterol Hepatol 1995; 7: $165-8$.

12 Calvet MC, Gresser I. Interferon enhances the excitability of cultured neurones. Nature 1979; 278: $558-60$.

13 Smith EM, Blalock JE. Human lymphocyte production of corticotropin and endorphin-like substances: association with leukocyte interferon. Proc Natl Acad Sci USA 1981; 78 $7350-4$. 